\title{
Vibration diagnostics of concrete block
}

\author{
Mariusz Żółtowski ${ }^{1,{ }^{* 1}}$, Michat Liss ${ }^{2}$, Jozef Melcer $^{3}$ \\ ${ }^{1}$ UTP University of Science and Technology in Bydgoszcz, Faculty of Management, Poland \\ ${ }^{2}$ UTP University of Science and Technology in Bydgoszcz, Faculty of Mechanical Engineering, \\ Poland \\ ${ }^{3}$ University of Zilina, Faculty of Civil Engineering, Slovakia
}

\begin{abstract}
Presented study indicates the possibility of using complex estimators of vibrational energy flow used in quality testing of concrete blocks, as part of a dedicated research methodology and detailed methodologies developed in many previous studies by the author. Theoretical analysis and practical verification of the sensitivity testing measures of complex vibration processes indicate the wide possibilities of their applications. Recognizing the need to improve the testing methods of building structures for assessing their degradation status - this paper presents significant results of the research procedure in the field of effectiveness verification of proposed mutual measures in wall element bench tests.
\end{abstract}

\section{Introduction}

Identification in building constructions becomes a method of assessing changes in the structure operational state, and the modal analysis methods with related vibration estimators well reflects the wall elements destruction. Searching for mapping the state of building material with the parameters of their vibration models, in bench tests and on real objects, made it possible to assess the similarity of models, the accuracy of decisions and the effectiveness of the used test method. Assessment of the building structures dynamic state with the help of vibration energy propagation measures requires the association of the structure features to assessed object with the set of measures and assessments of the initial processes.

Dedicated research procedure algorithm during vibrational energy flow analysis in the study of machines and building structures includes [9]:

- formulating a research problem;

- acquisition and processing of vibrational energy measures;

- qualitative optimization of vibration symptoms;

- models of binding measures with the degradation state;

- inference.

In general, the methodology for degradation state testing includes detailed procedures for the development of a data acquisition system, their processing and statistical inference repeatable in many different issues of vibration signals testing.

Measurements of vibrations along with their physical interpretation (the proposed model of the vibration signal passing through the material) constitute the basis for acquiring information in the study of selected masonry components degradation. Basic research in this work conducted to confirm the assumptions and conditions of specific destructive tests verify the validity of the adopted models and based on the recorded vibration signals characterize the dynamic properties of the tested materials. Elements of modelling theory and simulation research (necessary even in the properties recognition) allow to determine whether any research object (building material) in the simplified modelled with the

${ }^{\star}$ Corresponding author: mazolto@utp.edu.pl 
parameters $\mathrm{m}, \mathrm{k}, \mathrm{c}$ - a system with one degree of freedom - can be treated as a rigid system linear [7].

Considerations of this work indicate that the properties of such modelled object elements can be analysed by describing them with differential equations (solving the own problem) or the same information can be obtained by measuring vibrations ( $\mathrm{a}, \mathrm{v}, \mathrm{x}$ parameters of the vibration process) and on the basis procedure of determination vibration mutual measures $[1,2,9]$.

The detailed discussion of the vibration energy flow analysis and modal analysis methods indicates the development of software and measurement stations for the implementation of practically difficult measurement techniques in the non-dismantling assessment of entire building structures destruction status, or only in the research of structure elements, e.g. wall elements or segments.

Described in a definition and from the practical application of the vibration process measure (due to the process of averaging during their determination called estimators) indicate a large range of activities necessary during the acquisition and processing of information, activities programmed during the computer development and presentation of measurement data.

\section{Vibrations in degradation tests}

When researching real systems (constructions, structures, machines, devices), the basic problem is to determine the value of vibroacoustic energy stored, dispersed and transferred by individual elements of these systems. Knowledge of these quantities serves to assess material effort, fatigue, diagnostic tests and noise prediction, and facilitates the design of system components (eg vibroinsulation).

The development of measurement methods, especially measurements of energy quantities, significantly expanded the possibilities of sound radiation testing by structures and allowed to calculate the acoustic power radiated to the far field based on measurements in the near field. Methods of quantitative and qualitative research of vibroacoustic energy propagation in space with complex boundary areas have been developed. It related to the quantitative assessment of vibroacoustic energy accumulated in the construction elements and the assessment of energy radiated by these elements, as well as energy carried by various routes.

Fig. 1 presents basic (exemplary) waveforms of measured amplitude in time for subsequent measurements, which are the basis for further program studies for distinguishing information about state changes.
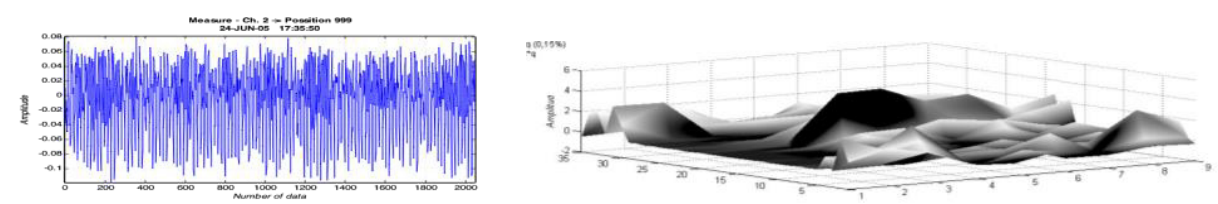

Fig. 1. Measurements of amplitude changes in time for subsequent vibration measurements.

The basic result of the time course measurement and its spectral analysis are shown in Fig.2. These are the main types of signal transformation used later in this work, because all other vibration signal characteristics are based on the above courses.

a)
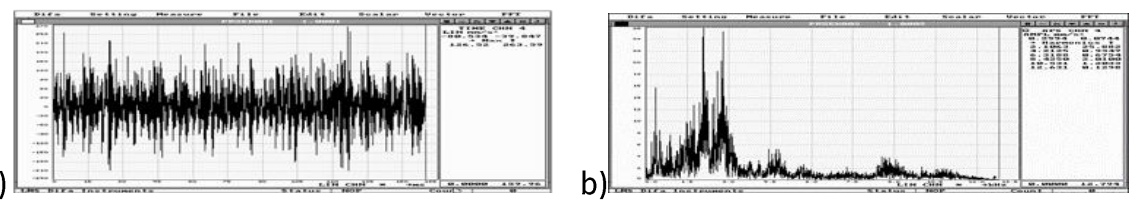

Fig. 2. Time course a). and the spectrum of the vibration signal b). 
Vibration processes are mostly accidental processes, that's why they are further processed in the processor according to different algorithms to obtain different types of signal estimators. These estimators, as opposed to signals, are already determined and depending on their type they reflect the various characteristics and information properties of the signals.

Estimators, as numerical measures of the vibration process, are defined in three categories of signal description: time, frequencies and amplitudes [1,9,12] and as determinate quantities reflecting the state of the evaluated material can be compared with the state standards of the tested materials.

The available literature $[1,4,7,8,9,11]$ presents the definitions and properties of various vibration estimators, distinguishing personal estimates - concerning one signal and mutual estimators - regarding two signals.

New tools in this area of research concern the possibilities of using the methodology to study the vibrational energy flow, as well as the modern acquisition and processing of vibration measures - to assess the quality of masonry elements, which form the basis for consideration in this work. In practical applications, they allow for a better understanding of the behavior of complex structures, optimization in the design process and assessment of dangerous states. In the latter area the content of the subject is placed, looking for measures to assess the state of destruction of new, long-term wall elements, and often with an unknown state of destruction and the wall's safety factor of many buildings.

The methodology for testing the vibrational energy flow used in the considerations of this work has been adapted from the dynamic field of mechanical structures. Its usefulness is greater for homogeneous structures of steel structures than for building materials such as masonry elements, where each test result is related to the structure, shape and physical state of the sample. Therefore, it is feared that the heterogeneity and coarse grain structure of concrete and ceramics may be an obstacle to the effectiveness of very precise measuring techniques. The task of this work is to assess the usefulness of measures of vibrational energy flow in the diagnosis of homogeneous masonry elements (bricks, blocks, blocks) and to develop recommendations for testing more heterogeneous masonry fragments.

The tasks of this work cover in detail the following issues: modeling and description of the process of destruction of masonry elements, the use of vibrations in the description of changes in their dynamic state; implementation of destructive testing of wall elements and segments, application of a software system for acquisition and processing of measurement data and statistical inference (SYMPTOMS GENERATE, VIOMA, OPTIMUM, SVD programs). The method of solving the main job includes methods, measures and procedures for: archiving the results of measurements of building materials programmed destructions, their processing aimed at selecting the best diagnostic measures and determining the relationship between the state of load (destruction) of the material tested and the values of measures.

The obtained results of the work carried out indicate the vibration measures characterizing the destruction of the tested wall elements, determined by a bench test method, based on measurements and processing of the vibration signal. They also assess wall elements destruction model and creates the basis for the construction of simple diagnostic procedures for the needs of the construction products control system placed on the market and for the assessment of the safety class of masonry walls.

These measures include $[3,5,8,10]$ :

- transmittance: the dynamics of the system in the frequency domain can be described by means of the equation

$$
X(j \omega)=H(j \omega) F(j \omega)
$$


where: $X(j \omega)$ is the vector of the system response spectra, $F(j \omega)$ is the vector of forcing forces spectra, $H(j \omega)$ is the frequency response matrix;

- FRF (spectral transition function): this is a frequency function that describes the relationship between the force of force $F(t)$ and the acceleration of vibrations $a(t)$ as the response signal. The frequency response function (FRF) can be described as the quotient of the Fourier transform value of the force that forces the oscillation $F(\omega)$ by the Fourier transform of the response signal $\mathrm{A}(\omega)$ :

$$
F R F=\frac{\sum F(\omega)}{\sum A(\omega)}
$$

The FRF function is a complex frequency function, the module of which presents the amplitude-frequency characteristic, whereas the argument is the angle of the phase shift between the forcing force and the response;

- coherence function: is a measure of the consistency of two oscillation processes $x(t)$ and $y(t))$ and is defined as follows $[1,4,7]$ :

$$
\gamma_{x y}^{2}(f) \equiv \frac{\left|G_{x y}(f)\right|^{2}}{G_{x x}(f) G_{y y}(f)}
$$

Regardless the transfer paths character of the transition paths, the coherence function always assumes a value of 1 , if only the signals $x(t)$ and $y(t)$ come from the same source. For more than one source, the non-negative coherence function is always less than one (from 0 to 1). The coherence function as a measure of the coherence of two signals has good diagnostic properties, because the appearance of a new fault signal violates the coherence of the previous ones, so that the coherence function decreases.

The new research tools developed in this work mainly concern the assessment of the applicability of the proposed vibration measures, modern vibration processing and statistical cause and effect reasoning in the study of selected building materials.

\section{Research methodology}

\subsection{Research object}

Identification tests as well as basic tests for randomly selected 6 concrete blocks were carried out on laboratory stands, deliberately adapted and suitably equipped. Providing a limited number of degrees of freedom is ensured by suspending the tested blocks on a thin line. Signal reception sensors were mounted on wax, and the directions of extortion were taken as shown in Fig. 3.
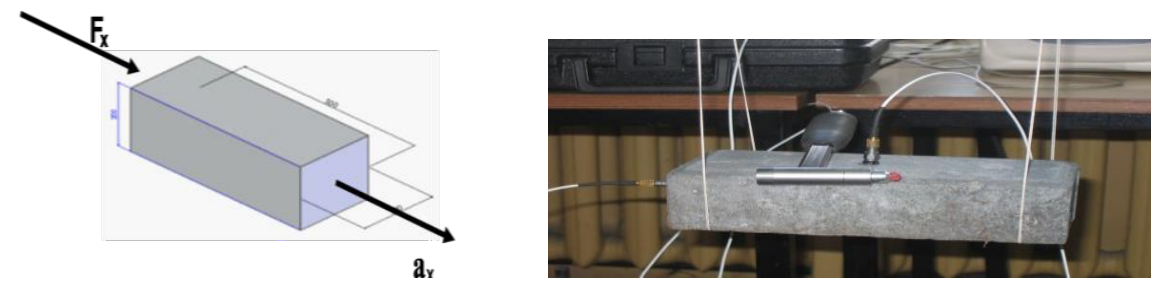

Fig. 3. Mounting of tested blocks and piezoelectric sensors.

The LMS TEST.XPRESS state-of-the-art measuring equipment was used to measure the time of force and response of the system as well as the determination of the H(f), FRF and coherence functions. This software enables to analyze the vibrational energy flow in elements and any other building constructions. 
The LMS TestXpress software allows for the recording of a forced signal and responses in the form of time courses and can generate mutual functions and their numerical values (area under the curve) used in the study of this work.

\subsection{Research position}

An interesting case of the conducted research of this work were the results obtained for a broken concrete block, with a distinct crack in the tested element, as shown in Fig.4.

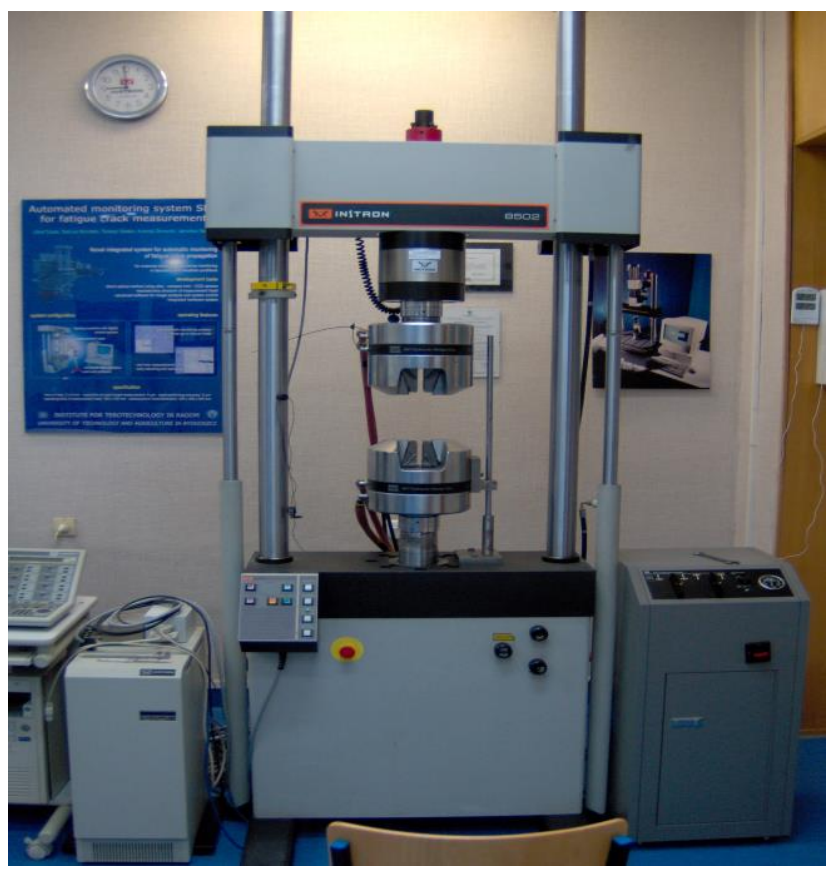

Fig. 4. INSTRON hydraulic press.

In the tests on the INSTRON 8502 testing machine, the range of the limit loads for the tested concrete blocks was determined for destruction, which allowed to determine the test loads for detailed tests. The destructive tests were carried out on 6 concrete blocks and after averaging the results, the values of loads for basic research were proposed.

\subsection{Findings}

Time passes of the force of force (its stability is ensured by the APB-200 system) and the corresponding vibration acceleration courses for the group of 6 concrete blocks tested are shown, for example, in the coordinate system: acceleration - time in Fig. 5 for elements without a destructive load. Similar analyzes were recorded for further processing for vibration-inducing forces, as well as for the loads highlighted on the Instron machine.

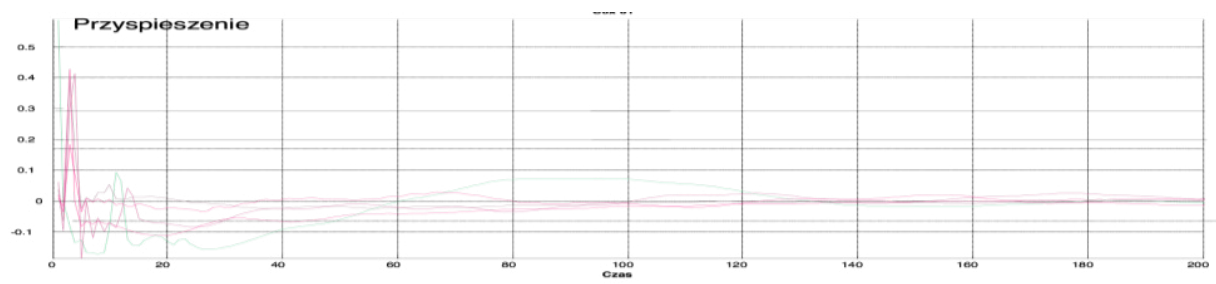

Fig. 5. Amplitudes of vibration acceleration of the tested block for various F0x loads. 
The numerical values of the vibration signal measures for the tested concrete block, with various strength loads recommended in the tests of this work, are presented in Table 1.

Table 1. Measurement data of pre-selected measures.

\begin{tabular}{|c|c|c|c|c|c|c|c|c|c|}
\hline $\begin{array}{c}\text { Wartość } \\
\text { siły }\end{array}$ & FRFr & FRFu & $\mathbf{H}(\mathbf{f}) \mathbf{r}$ & $\mathbf{H}(\mathbf{f}) \mathbf{u}$ & coher & delta & Urms & w1 & w2 \\
\hline 0 & 1824,87 & 40,32 & 20,8 & 1,57 & 179,48 & 0,916 & 0,011 & 4 & 40 \\
\hline 50 & 1115,24 & $-1502,55$ & 8,56 & 22,56 & 107,01 & 0,992 & 0,011 & 2 & 40 \\
\hline 100 & 326,82 & $-340,92$ & $-3,01$ & 24,57 & 92,48 & 0,969 & 0,011 & 2 & 30 \\
\hline 150 & $-1084,96$ & $-397,68$ & $-14,48$ & 21,46 & 88,45 & 0,959 & 0,011 & 2 & 50 \\
\hline
\end{tabular}

In the following drawings, the modes of forced force and the corresponding amplitudes of vibration accelerations for the tested wall elements are presented with the loads tested F1, F2, F3, respectively, specified in table 1. It is worth noting that the tested concrete blocks were loaded during the force tests: $50,100,150 \mathrm{kN}$.

Considering all the conducted sensitivity analyzes, using different statistical methods used in this work, it is possible to further propose as the main following measures of concrete block destruction state:

$\mathrm{FRF}_{\mathrm{r}}, \quad \mathrm{FRF}_{\mathrm{u}}, \quad \mathrm{H}(\mathrm{f})_{\mathrm{r}}, \quad \mathbf{H}(\mathbf{f})_{\mathrm{u}}, \mathrm{U}_{\mathrm{RMS}}, \quad$ coher, $\omega_{2}$.

Because of the tests carried out for the indicated loads along the $\mathrm{Z}$ axis, the results presented in Fig. 6 were obtained.
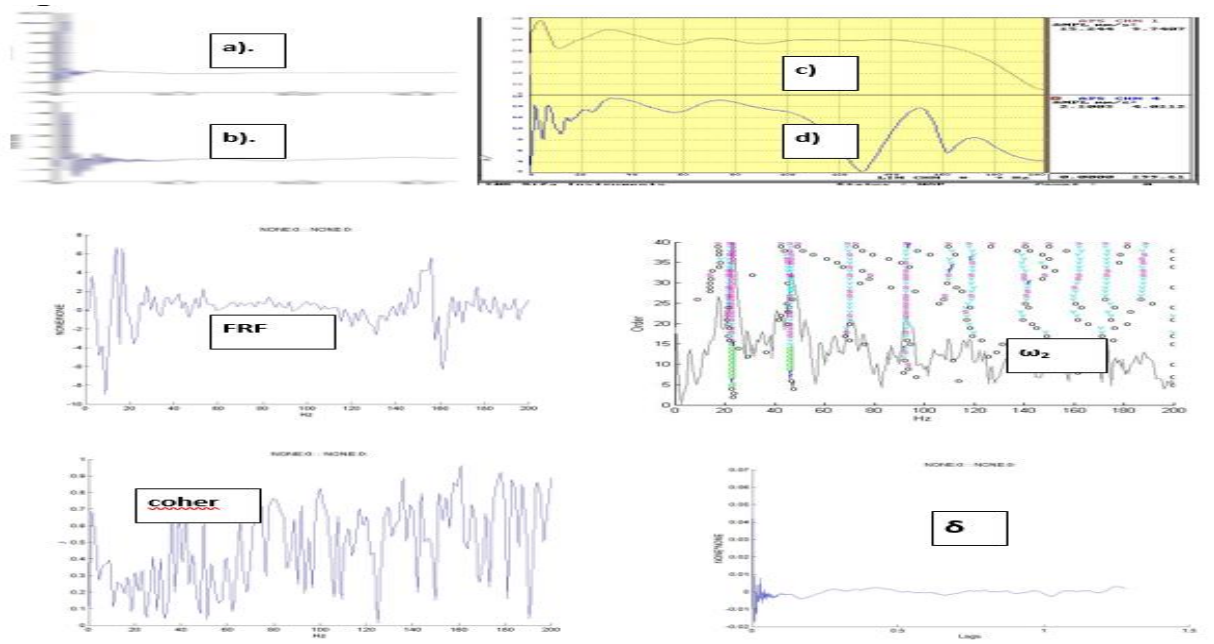

Fig. 6. X-axis test results (no load) - cracked block.

Fig. 6 shows the courses of force (a), its vibration acceleration amplitude (b), vibration spectrum of these signals (c, d) and exemplary measures of destruction: FRF function, values of natural frequency from the stabilization diagram with the characteristic frequency $\boldsymbol{\omega}_{2}$, coherence function (coher) and decay indicator of the mutual correlation function $\boldsymbol{\delta}$. The differences in the obtained measures for the broken block in relation to the undamaged one are clearly visible, which is mainly manifested by the appearance of the second natural frequency around $150 \mathrm{~Hz}$ - (see Fig. 6.d), FRF and $\omega 2$.

All functional waveforms were further developed (discretized) numerically and used during qualitative inference using the previously proposed statistical methods $[7,9,10]$.

Differentiation of vibration state measures for a fit and damaged pulley in the study of this work is presented in the collective drawing in the form of stabilization diagrams and field values under the curves of the FRF and Coherence functions 

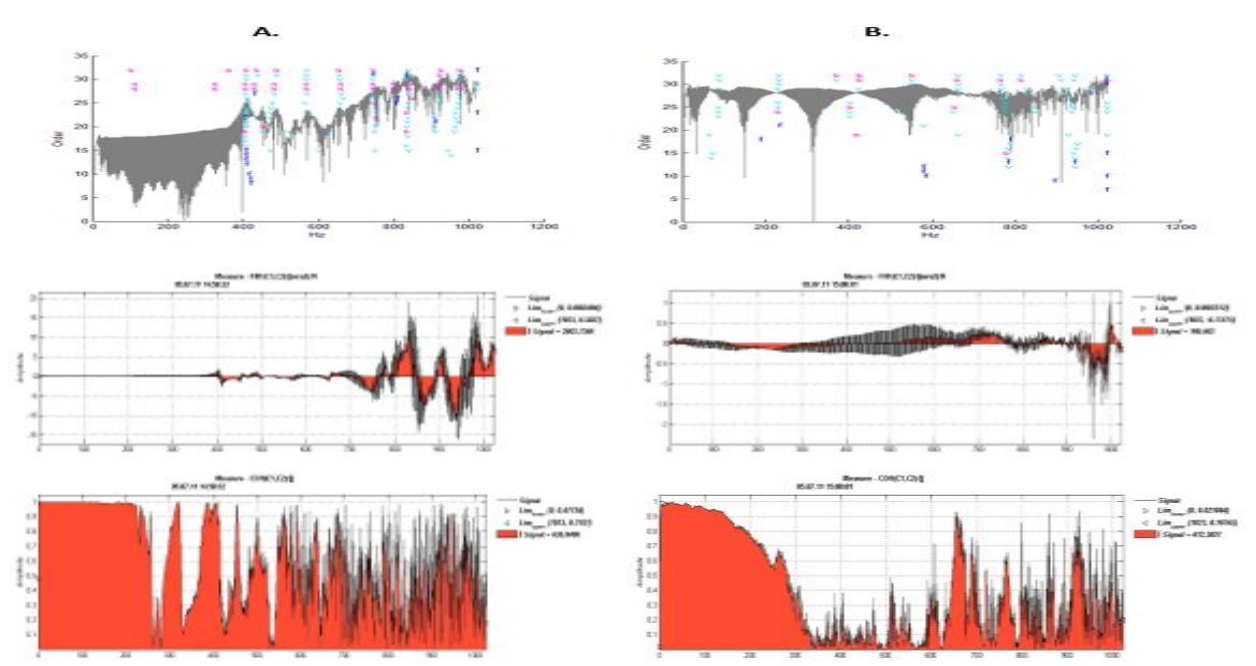

Fig. 7. Stabilization diagrams, FRF and Coherence functions for a suitable (A.) and damaged concrete block (B.).

The use of all data, usually collected in excess in the experiment and obtained during processing, to decide about the state of destruction of a masonry element is often neither possible nor necessary. It is natural, therefore, to strive to reduce the initial data, including the data sets of the examined objects and information describing them, which should be replaced by relatively small sets of data respectively aggregated and ordered. In general, such data reduction may consist of:

- information selection, i.e. a reduction in the number of measures describing the materials tested;

- replacing the continuous range of variability of the measure with discrete and sometimes binary changes;

- reduction in the number of materials tested representing individual status classes.

The reduction methods are of great importance for the problem of information selection, which:

and). they minimize the risk or average probability of classification error of the object;

b). maximize the assumed objective function;

c). they maximize (with limitation) information indicators of features.

The optimal reduction methods (a) minimize the average risk or average probability of error in the classification of the object's condition. Practically, the importance of these methods can be significantly reduced due to ignorance of probability distributions. In such a situation, at least some examples of observations from individual classes must be known a priori. Types of data reduction b) and c) fall within the scope of methods used in mathematical statistics and methods of sampling and quantizing signals aiming at adapting the signal form to the requirements related to information processing.

In terms of the selection of information, the procedures of OPTIMUM, SVD, and SIBI recommended in many other studies [13]. The use of functional measures in the processing has been replaced by numerical measures, proposing determining the values of the functional waveform with the numerical value of the field under the curve, as shown in Fig.7.

The implementation of algorithms for the indicated methods illustrated in Fig. 8, allows distinguishing information-related vibration measures used to build a cause-and-effect model that forms the basis of the proposed assessment procedure for the degradation of the tested concrete block. 
The next steps of the calculation procedure in the OPTIMUM method are presented in the table $2[6,9,13]$.

Table 2. Vibration measures processed in the OPTIMUM [9] procedure.

\begin{tabular}{|c|c|c|c|c|c|c|c|c|c|}
\hline & FRFr & FRFu & $H(f) r$ & $H(f) u$ & coher & delta & Urms & w1 & w2 \\
\hline f1 & 2,287 & 1,207 & 5,108 & 0,611 & 0,364 & 0,033 & 0,000 & 0,400 & 0,204 \\
\hline $\begin{array}{r}\max _{\text {(f1) }} \\
\text { (1) }\end{array}$ & 5,108 & & & & & & & & \\
\hline f1 ${ }^{*}$ & 0,448 & 0,236 & 1,000 & 0,120 & 0,071 & 0,006 & 0,000 & 0,078 & 0,040 \\
\hline$f 1^{* *}$ & 1,000 & & & & & & & & \\
\hline f2 & 0,985 & 0,030 & 1,000 & 0,743 & 0,874 & 0,430 & 0,001 & 0,775 & 0,316 \\
\hline $\begin{array}{r}\max \\
\text { (f2) }\end{array}$ & 1,000 & & & & & & & & \\
\hline f2* & 0,985 & 0,030 & 1,000 & 0,743 & 0,874 & 0,430 & 0,001 & 0,775 & 0,316 \\
\hline$f 2^{* *}$ & 1,000 & & & & & & & & \\
\hline Li & 0.553 & 1,235 & 0,001 & 0.917 & 0.937 & 1.145 & 1.414 & 0,949 & 1.179 \\
\hline $1 / \mathrm{Li}$ & 1,810 & 0,810 & 1000 & 1,090 & 1,067 & 0,873 & 0,707 & 1,054 & 0,848 \\
\hline wi & 0,002 & 0,001 & 0,992 & 0,001 & 0,001 & 0,001 & 0,001 & 0,001 & 0,001 \\
\hline
\end{tabular}

The algorithm of processing the measures in the graphic interpretation for the OPTIMUM method is shown in Fig.8A, and the result of the statistical elaboration in the SVD procedure in Fig.8B.
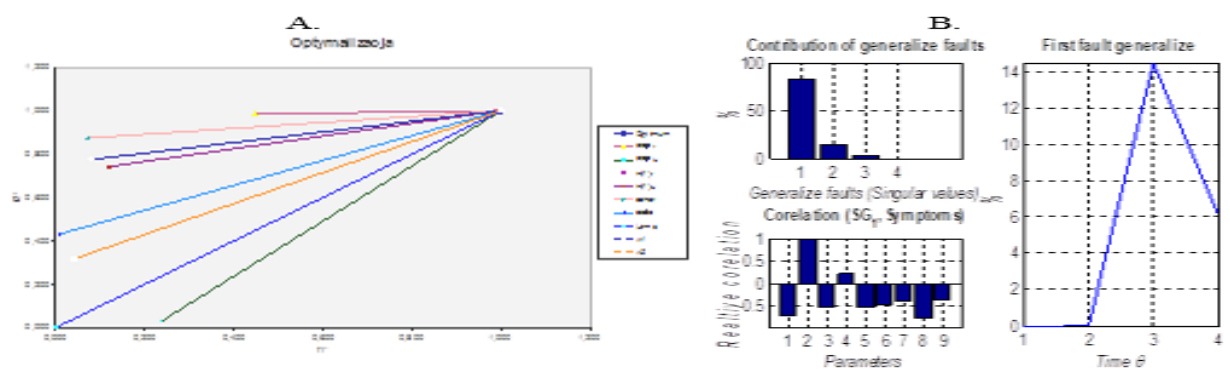

Fig. 8. Graphical presentation of sample results from OPTIMUM and SVD.

A further procedure in accordance with the methodology proposed for the study of vibration energy distribution proposed in the author's work was to determine good measures of the state of destruction of the concrete blocks tested and to establish cause and effect relationships between the state of block destruction and its measures. This was obtained by examining the functional relationship separately for each variable, i.e. for each measure individually characterizing the state of destruction and when testing the function of several variables, for all measures simultaneously. The study of these mathematical relations was carried out using simple regression and multiple regression, and the corresponding equations are shown directly on the regression curve drawings.

For the distinguished measures of degradation status, regression partial models were determined, for example shown in Fig.9. To approximate the form of the function between the state of destruction and the measures of this state, the most preferred algebraic polynomials in the statistics, with the especially recommended second-degree polynomial, were adopted. The obtained relations indicate how many changes in the load of the tested wall elements affect changes in the values of the vibration signal selected in the examination procedure (individual or multiple). 
Measurements of approximation errors of the examined functions were determined by the multidimensional correlation coefficient R2, also called the coefficient of determination, taking values from the interval [0,1]. Its values are given in regression drawings, the higher its value, the better the correlation - better adjustment of the regression curve to the measurement results.
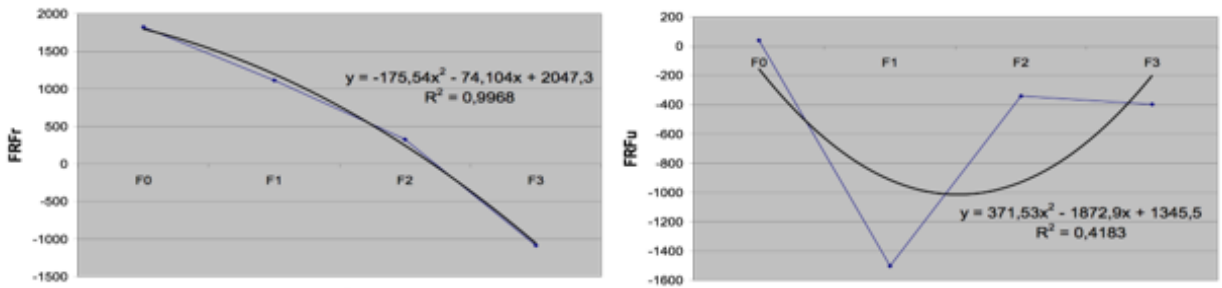

Fig. 9. Partial models for the indicated vibration measures

The expected effect of data reduction for the adopted quality of state classification is to simplify the structure of the data set, simplify the classification algorithm, shorten the calculation time, increase the speed of the system operation, reduce technical implementation costs.

Finally, the state degradation measures for a concrete block are: FRFr, H(f)r, coher. used in accordance with the model shown in Fig. 10.
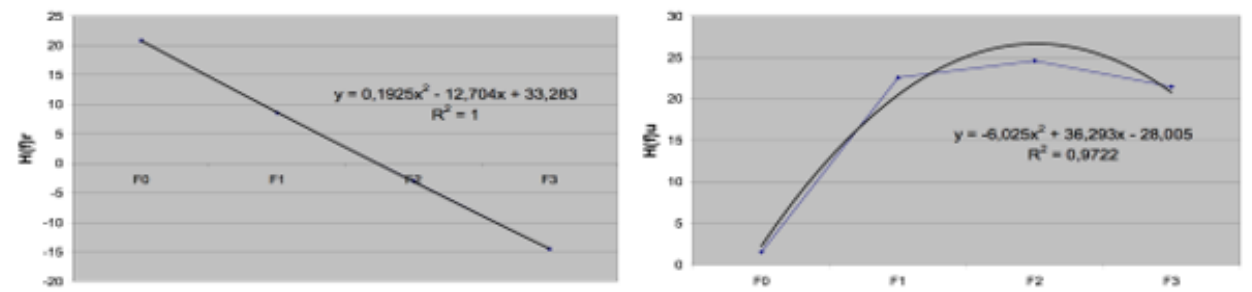

Fig. 10. The resulting cause and effect model for the tested concrete blocks.

The developed model for investigations of concrete block degradation that captures measures well differentiating the state of destruction is the basis for building a test method useful in industrial production.

\section{Summary}

Vibrations measurements along with their physical interpretation form the basis of the information obtained in the study of the destruction of selected building structures or their masonry components.

The exemplification of the method on a group of concrete blocks carried out in this study confirmed the compliance and effectiveness of the proposed dedicated methodology developed for the study of degradation of materials and structures in construction. The assumptions of the developed research methodology were positively verified and the effectiveness of complex vibration measures in the application for the assessment of degradation of masonry materials was confirmed, which promises well for the needs of real-life research on long-term objects.

The considerations of this study confirm the usefulness of the proposed algorithms and programs supporting the implementation of the research methodology, that is: the acquisition and processing of information, the reduction of excess information, statistical inference and the presentation of the study results.

The bursting of the block can be located by means of the coherence function (which already has an indication in the mathematical record for energy evaluation between points) and mutual measures: transmittance and its inversion - FRF. 
Examining the structure state or its elements thanks to vibration measurements (acceleration a, velocity $\mathrm{v}$, displacement $\mathrm{x}$ ), can predict and prevent the possible failure time and prevented it by proper repair. What is more, by tracking the amplitudes of individual spectral components or other measures of the vibration process that constitute the destruction of elements, their condition, so important for the correct exploitation of long-term building structures, can be assessed without any problems.

\section{References}

1. B. Żółtowski, L. Castaneda, Structural diagnosis of rail vehicles and method for redesign. Diagnostyka Vol.15 (2014).

2. B. Żółtowski, M. Łukasiewicz, Diagnostyka drganiowa maszyn. (ITE-PIB 2012).

3. B. Żółtowski, M. Łukasiewicz, T. Kałaczyński, Techniki informatyczne $w$ badaniach stanu maszyn (UTP Bydgoszcz 2012).

4. B. Żółtowski, M. Żółtowski, Vibration signals in mechanical engineering and construction (ITE-PIB 2015).

5. B. Żółtowski, C. Cempel, Engineering of diagnostics machines (PTDT, ITE - PIB 2004).

6. M. Żółtowski, Operacyjna analiza modalna $w$ badaniach konstrukcji budowlanych (WU UTP Bydgoszcz 2012).

7. M. Żółtowski, Investigations of harbour brick structures by using operational modal analysis. Polish Maritime Research No 1, (2014).

8. M. Żółtowski, B. Żółtowski, Vibrations signal to the description of structural damage of dynamic the technical systems (XIII International Technical Systems Degradation Conference 2015).

9. M. Żółtowski, Investigations of harbour brick structures by using operational modal analysis. Polish Maritime Research No. 1/(81) vol.21 (2014).

10. M. Żółtowski, M. Liss, The use of modal analysis in the evaluation of welded steel structures. Studies and Proceedings of Polish Association for Knowledge Management Vol.79 (2016).

11. M. Żółtowski, M. Liss, Zastosowanie eksperymentalnej analizy modalnej $w$ ocenie zmian sztywności prostego elementu konstrukcyjnego. Studies and Proceedings of Polish Association for Knowledge Management Vol. 80 (2016).

12. M. Żółtowski, R.M. Martinod, Technical Condition Assessment of Masonry Structural Components using Frequency Response Function (FRF). Masonry International Journal of the International Masonry Society Vol.29,No.1 (2016).

13. M. Żółtowski, R.M. Martinod, Quality identification methodology applied to wallelements based on modal analysis. Civil Engineering the Athens Institute for Education and Research, Emerald, Athens (2015). 